\title{
Satisfaction with the Effect of Local Dynamical Micro- massage Therapy on the Pain and Discomfort after Breast Reconstruction Surgery
}

\author{
Kwang Hyeon Ahn \\ Sun Jae Lee \\ Eun Soo Park \\ Yu Gil Park
}

Department of Plastic \& Reconstructive Surgery, Soonchunhyang University Bucheon Hospital, Bucheon, Korea
Received December 27, 2019

Accepted January 7, 2020

\footnotetext{
Correspondence

Eun Soo Park

Department of Plastic and Reconstructive Surgery, Soonchunhyang University Bucheon Hospital, 170 Jomaru-ro, Bucheon 14584, Korea Tel.: +82-32-621-5319

Fax: +82-32-621-5016

E-mail: peunsoodschmc.ac.kr

(C) Korean Society for Laser Medicine and Surgery

(c) This is an open access article distributed under the terms of the Creative Commons Attribution NonCommercial License (http://creativecommons.org/ licenses/by-nc/4.0) which permits unrestricted noncommercial use, distribution, and reproduction in any medium, provided the original work is properly cited.
}

\author{
Background and Objectives \\ Breast reconstruction has the advantage of reducing the loss of the body \\ image of patients after mastectomy surgery, and also improving the \\ quality of the social and sexual life of breast cancer patients. However, in \\ surprising and unfortunate number of patients, acute postoperative pain \\ persists beyond the normal course of postsurgical recovery. We set out \\ to investigate the effect of local dynamical micro-massage (LDM) \\ treatment for achieving pain relief and reducing other postoperative \\ complications.
}

\begin{abstract}
Materials and Methods
We performed a retrospective analysis on 58 patients who underwent LDM treatment for postoperative pain management at Soonchunhyang University Bucheon Hospital between February 2017 and June 2019. Those patients who complained of persistent postoperative pain, which was uncontrollable with medication, were treated with LDM. The degree of pain and discomfort with contracture were recorded using numerical rating scale (NRS) scoring system with numbers from 0 to 10 ('none' to 'worst').
\end{abstract}

\section{Results}

The median NRS score of pain was reduced by $62.3 \%$ from the start to the end of LDM treatment ( $p<0.001)$. Further, the NRS score of discomfort with contracture was reduced $66.0 \%(p<0.001)$. There was no complication related to the LDM treatment.

\section{Conclusion}

Dual-frequency ultrasound LDM can be an effective therapeutic option for persisting pain after breast reconstruction surgery. It was also effective in improvement of discomfort with contracture and erythema of the surgical wound.

\section{Key words}

Dual-frequency ultrasound; Local dynamical micro-massage; Postoperative pain; Contracture; Breast reconstruction 


\section{INTRODUCTION}

Nowadays, as people are concern with their quality of life, reconstruction of breast is considered essential for breast cancer patients. Breast reconstruction has the advantage of reducing the loss of body image of patient after mastectomy surgery and improving the quality of social and sexual life of breast cancer patients.' In Korea, the National Health Insurance Service (NHIS) began to cover breast reconstruction after total mastectomy in April 2015. ${ }^{2}$ With the expansion of insurance for breast reconstructive surgery, more reconstructive surgery is expected to be performed. In addition to the increased interest in such reconstructive surgery, there is an increasing concern about the complication of reconstructive surgery. There are many complications; seroma and hematoma collection, infection, skin necrosis, loss of sensation, contracture, etc. Among these complications, the post-operative pain is most common and annoying one to the patients. For the most of patients, acute postoperative pain after reconstruction surgery is an expectable surgical outcome, and it usually resolves with time and appropriate medication. ${ }^{3}$ However, in surprising and unfortunate number of patients, acute postoperative pain persists beyond the normal course of postsurgical recovery. ${ }^{4}$ These patients report less satisfaction with the reconstruction surgery, even though the aesthetic outcomes were satisfactory. Also, the prolonged acute postoperative pain can be a risk factor for the development of chronic pain syndrome. ${ }^{5}$ So, it is clinically important to manage the early acute postsurgical pain with aggressive methods. Various agents (opioid vs. non-opioid), routes (oral, intravenous, neuraxial, regional) and modes (patient controlled vs. "as needed") for the treatment of postoperative pain exist. ${ }^{6}$

Also, therapeutic ultrasound treatment has been used for the 40 years to treat soft tissue injury. ${ }^{7}$ Ultrasound therapy is widely applied in various musculoskeletal disorders such as joint contracture, scar tissue, and tendinitis, and it is known to increase extensibility of collagen tissues and raise pain thresholds. ${ }^{8}$ We had clinical experience of using local dynamical micro-massage $\left(\right.$ LDM $^{\circledR}$ MED; Wellcomet, Karlsruhe, Germanyl for radiationinduced fibrosis in a breast cancer patient. After 12 times of LDM treatments, the NRS score was decreased from 6 to $2 .{ }^{9}$ With this clinical experiences, we used LDM treatment for the postoperative pain in breast reconstruction patients. In the following study, we set out to investigate the effect of LDM treatment in pain relief and other postoperative complications.

\section{MATERIALS AND METHODS}

\section{Patients}

We performed a retrospective analysis on 58 patients who underwent LDM treatment for postoperative pain management at Soonchunhyang University Bucheon Hospital between February 2017 and June 2019. The inclusion criteria for this study were as follows: patients who had uncontrollable postoperative pain after unilateral or bilateral breast reconstruction with latissimus dorsi myocutaneous (LD) flap and transverse rectus abdominis myocutaneous (TRAM) flap. The data were collected from the database and medical records of the patients. Written consents were obtained from each patient for both the laser treatment and the publication of photographs of the results.

\section{LDM treatment protocol}

Patients who complained persistent postoperative pain, which was uncontrollable with medication, were treated with LDM (Fig. 1A) daily or every other day. The Ulcus cruris mode of LDM dermatologic program was used in

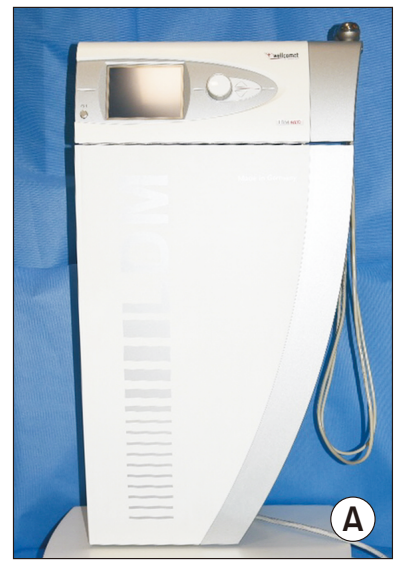

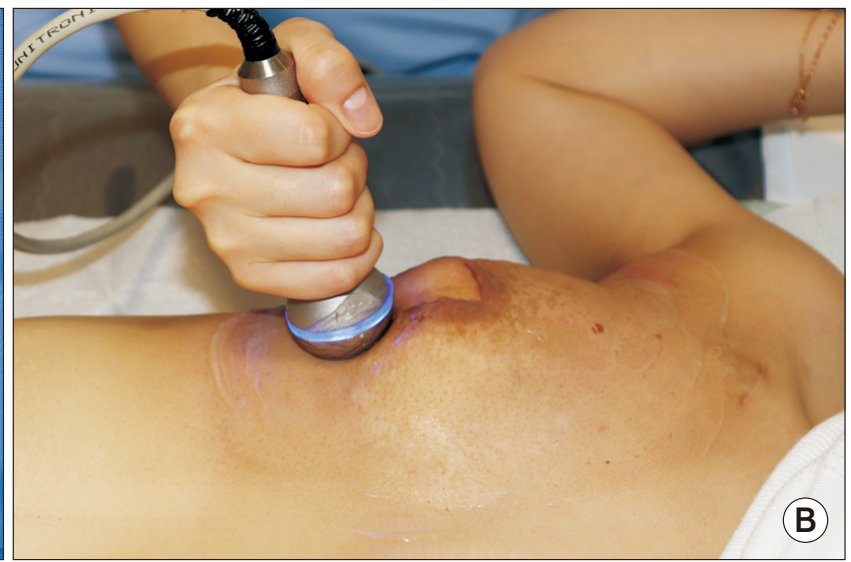

Fig. 1. (A) LDM-MED ${ }^{\circledR}$ (Wellcomet, Karlsruhe, Germany). (B) The Ulcus cruris mode of LDMMED $^{\circledR}$ dermatologic program was used in treatment. 
treatment. The protocol of this mode was set as $10 \mathrm{MHz}$ with intensity of $1.5 \mathrm{~W} / \mathrm{cm}^{2}$ and $1.0 \mathrm{~W} / \mathrm{cm}^{2}$ for 2 minutes each, then was followed by $3 / 10 \mathrm{MHz}$ dual frequency setting with intensity of $1.0 \mathrm{~W} / \mathrm{cm}^{2}$ and $2.0 \mathrm{~W} / \mathrm{cm}^{2}$ each for 3 minutes (Fig. 1B).

\section{Evaluation of outcomes}

At the beginning and end of the LDM treatment, the patients were asked to evaluate their degree of pain, discomfort with contracture with NRS scoring system with numbers from 0 to 10 ('none' to 'worst').

\section{Statistical analysis}

Statistical analyses were performed using SPSS version 26.0 (IBM Corp., Armonk, NY, USA). The Wilcoxon signed rank test was used to compare the patients' NRS scores of pain and discomfort with contracture before and after LDM treatment. Statistical significance was considered to be $p<0.05$.

\section{RESULTS}

\section{Patient characteristics}

Total 58 patients were treated with LDM for persisting pain after breast reconstruction surgery. As shown in Table 1, the mean age of patients was 48.8 years (range, 2972) and the mean LDM cycle was 17.8 cycle (Range, 2-46). In the type of reconstruction, most of the cases were latissimus dorsi myocutaneous flap with insertion of silicone implant. Two cases were transverse rectus abdominis myocutaneous flap, and 5 cases were reconstructed with only silicone implant. In the site of LDM treatment, 25 cases were left breast, 28 cases were right breast. 2 patients were treated in both breast, and the donor site pain cases were 3.

Table 1. Demographic Characteristics of 58 patients of LDM treatment

\begin{tabular}{lc}
\hline Characteristic & Value \\
\hline Female & 58 \\
Mean age, $y$ (range) & $48.8(29-72)$ \\
Reconstruction type & 51 \\
LD flap + implant & 2 \\
TRAM flap & 5 \\
DTI & \\
LDM site & 25 \\
Left breast & 28 \\
Right breast & 2 \\
Both breast & 3 \\
Donor site & $17.8(2-46)$ \\
Mean LDM cycle &
\end{tabular}

The NRS score were available for all 58 patients at both the start and the end of LDM treatment. As shown in the Fig. 2, median NRS score of pain was reduced by $62.3 \%$ from the start to the end of LDM treatment $(p<0.001)$. Also the NRS score of discomfort with contracture was reduced $66.0 \%(p<0.001)$. There was no complication related to the LDM treatment.

\section{DISCUSSION}

Pain is defines as an unpleasant sensory and emotional experience associated with actual or potential tissue damage, or described in terms of such damage according to The International Association for the Study of Pain's. ${ }^{10}$ More than $80 \%$ of patients experience acute postoperative pain after surgical procedure, and approximately $75 \%$ of patients with postoperative pain report the severity of pain as moderate, severe, or extreme. ${ }^{11,12}$ With underestimation of importance of aggressive pain control, many patients develop chronic pain after surgery. ${ }^{13}$ Mechanism of postoperative pain with surgical incision can be understood in 3 path way; Spinal sensitization, peripheral sensitization, and neuroplastic change in brain. ${ }^{14}$ In patients of breast reconstruction surgery, younger age, bilateral reconstruction, and severity of preoperative pain, anxiety, and depression were found as the factors associated with more severe acute postoperative pain. ${ }^{4}$

Medical ultrasound was first developed by Dr. George Ludwig at the Naval Medical Research Institution in the late 1940s, and there have been many attempts to use it in various medical field. In 1942, Lynn and Putnam successfully used ultrasound waves to destroy brain tissue in animals. It was considered as the earliest experiment of

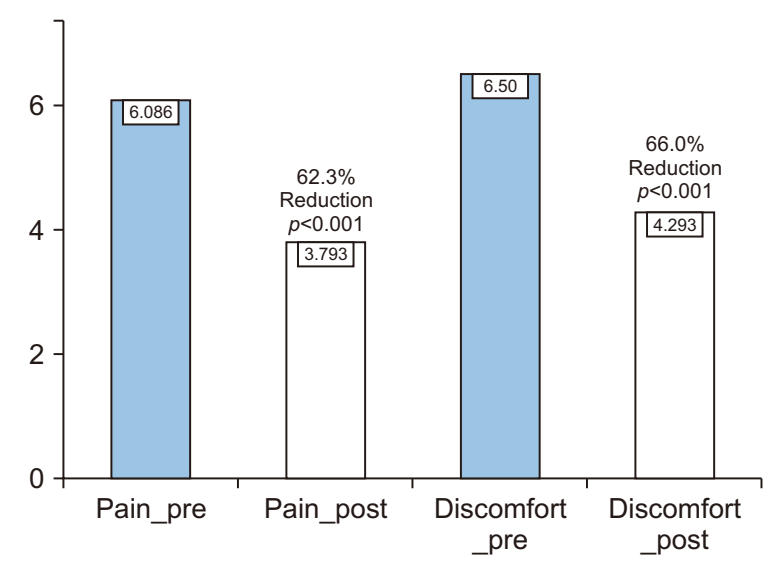

Fig. 2. Changes in median NRS score of Pain and Discomfort with contracture from the start of Local dynamical micro-massage (LDM) to the end of LDM. p-values from Wilcoxon signed rank test. 
therapeutic ultrasound application on biological tissues. ${ }^{15}$ Therapeutic effect of ultrasound is related in tissue healing in two different mechanism; thermal and non-thermal. In thermal mode, it can be used to selectively raise the temperature of tissue by increasing molecular vibration causing heat generation. ${ }^{16}$ In non-thermal aspect, the cavitation and acoustic streaming effect are considered as the main mechanism. ${ }^{17}$ In field of pain control with therapeutic ultrasound, the mechanism of pain relief is assumed as the followings: (i) elevation of tissue temperature leading vasodilation and acceleration of lymph flow; (ii) increased cell membrane permeability and improved gas exchange, and (iii) reduced conductivity of insonated nerves with resultant decrease in pain transmission. The increased blood flow also helps the resolution of chronic pain. ${ }^{7}$

Local dynamical micro-massage is the dual frequency therapeutic ultrasound. In the dual-frequency of LDM wave, the frequency is not constant, but oscillates between two frequencies; 3 and $10 \mathrm{MHz}$. This oscillation in every $5 \mathrm{~ms}$ is effective in decreasing MMPs and to stimulate HSPs, helping wound healing process. ${ }^{18}$ This presents the unique possibility to produce a dynamic modulation of the LDM-induced micro-massage effect. ${ }^{19}$ Irene Tausch et al. in 2015, also demonstrated the benefit of LDM treatment in reducing the level of pain after injection lipolysis. ${ }^{20}$ In our study, the patients received 2 to 46 times of LDM treatments for the postoperative pain which was not controlled with conventional medication. We found the significant improvement of their pain and discomfort with contracture after series of LDM treatments.

There was several limitation in our study. First, the pain and discomfort score was subjective method of evaluating the patients' status. More objective description of their status would be required for the further study. Second, the study was retrospective design for the pain evaluation with medical chart review. Despite these limitations, this study showed there was significant improvement of patients' pain and discomfort, and LDM could be an alternative method of controlling postoperative pain after breast reconstruction surgery.

\section{CONCLUSION}

Dual-frequency ultrasound LDM can be an effective therapeutic option for persisting pain after breast reconstruction surgery. It was also effective in improvement of discomfort with contracture and erythema of surgical wound. Further study could be designed to compare the effect with other conventional pain control methods.

\section{ACKNOWLEDGEMENTS}

This work was supported by the Soonchunhyang University Research Fund.

\section{CONFLICT OF INTEREST}

The authors declare that they have no conflicts of interest to disclose.

\section{REFERENCES}

1. Guyomard V, Leinster S, Wilkinson M. Systematic review of studies of patients' satisfaction with breast reconstruction after mastectomy. Breast 2007;16:547-67.

2. Ministry of Health and Welfare. Details on the criteria and method of application of allowance of medical care_revision [Internet]. Sejong: Ministry of Health and Welfare [cited 2018 Mar 27]. Available from: http://www.mohw.go.kr/react/index. jsp.

3. Ducic I, Seiboth LA, lorio ML. Chronic postoperative breast pain: danger zones for nerve injuries. Plast Reconstr Surg 2011;127:41-6.

4. Kulkarni AR, Pusic AL, Hamill JB, Kim HM, Qi J, Wilkins EG, et al. Factors associated with acute postoperative pain following breast reconstruction. JPRAS Open 2017;11:1-13.

5. Kehlet $H$, Jensen TS, Woolf CJ. Persistent postsurgical pain: risk factors and prevention. Lancet 2006;367:1618-25.

6. Garimella V, Cellini C. Postoperative pain control. Clin Colon Rectal Surg 2013;26:191-6.

7. Hashish I, Hai HK, Harvey W, Feinmann C, Harris M. Reduction of postoperative pain and swelling by ultrasound treatment: a placebo effect. Pain 1988;33:303-11.

8. Michlovitz SL. Thermal agents in rehabilitation. Philadelphia: Davis; 1990.

9. Choi YS, Park ES. Application of dual-frequency ultrasound to radiation-induced fibrosis in a breast cancer patient. Med Lasers 2017;6:86-9.

10. Bonica JJ. The need of a taxonomy. Pain 1979;6:247-8.

11. Apfelbaum JL, Chen C, Mehta SS, Gan TJ. Postoperative pain experience: results from a national survey suggest postoperative pain continues to be undermanaged. Anesth Analg 2003;97:534-40.

12. Gan TJ, Habib AS, Miller TE, White W, Apfelbaum JL. Incidence, patient satisfaction, and perceptions of post-surgical pain: results from a US national survey. Curr Med Res Opin 2014;30:149-60.

13. Fletcher D, Stamer UM, Pogatzki-Zahn E, Zaslansky R, Tanase NV, Perruchoud C, et al. Chronic postsurgical pain in Europe: an observational study. Eur J Anaesthesiol 2015;32:725-34. 
14. Pogatzki-Zahn EM, Segelcke D, Schug SA. Postoperative painfrom mechanisms to treatment. Pain Rep 2017;2:e588.

15. Dénier A. Les ultra-sons appliqués à la médecine. Paris: L'Expansion scientifique française; 1951.

16. Watson T. Electrotherapy: evidence-based practice. Elsevier Health Sciences; 2008.

17. Baker KG, Robertson VJ, Duck FA. A review of therapeutic ultrasound: biophysical effects. Phys Ther 2001;8:1351-8.

18. Choi YS, Nam SM, Park ES. Clinical experience of an effective treatment of intractable chronic venous ulcer on the lower leg using dual-frequency ultrasound. Med Lasers 2017;6:29-31.

19. Lee WS, Park ES, Kang SG, Tak MS, Kim CH. Successful treat- ment of recalcitrant remaining postoperative wounds by dualfrequency ultrasound. Med Lasers 2018;7:74-8.

20. Tausch I, Kruglikov I. The benefit of dual-frequency ultrasound in patients treated by injection lipolysis. J Clin Aesthet Dermatol 2015;8:42-6.

How to cite this article: Ahn KH, Lee SJ, Park ES, Park $Y G$. Satisfaction with the effect of local dynamical micromassage therapy on the pain and discomfort after breast reconstruction surgery. Med Lasers 2020;9:39-43. https://doi. org/10.25289/ML.2020.9.1.39 